\title{
A Pulse-Compression Method for Process Monitoring ${ }^{1}$
}

\author{
N. Eva Wu and Xiaoxia 'Wang \\ Department of Electrical Engineering, Binghamton University, Binghamton, NY 13902-6000 \\ evawu, bg23823@binghamtisn. edu
}

\begin{abstract}
This paper describes, from a deterministic view point, an on-line process monitoring method for small signal bchavior of time invariant stable systems at a given opcrating point. The monitoring is acisomplished by using an m-sequence modulated low-pass wavelet as the probing signal, and a cyclic reference signal as the correlator. This method allows to achicve high resolution, high signal to noise ratio monitoring. and the acquired data can be used for on-line diagnosis.
\end{abstract}

\section{Introduction}

Health monitoring and diagnosis of industrial processes provide situational awareness and fredback information that help optimize operational effectiveness, prevent catastrophic failures, and decrease maintenance costs. Existing approaches to process monitoring include model-bascd, knowledge-based, and multivariable statistical approaches[8]. This paper applies the concept of pulse-compression probing to process monitoring. In this casc, a compressible finite duration probing signal stimulates a process The response is processed by a corrclator with a cyclic reference signal which compresses the response of the probing signal into a narrow source wavelet, called the compressed version of the probing signal. Functionally the compressed version of the probing signal equivalently excites the process to yicld the probing output from which the impulse response that reflects the small signal characteristics of the process can be extracted. Deviation of the impulse response from its normality is an indication of abnormality of the process. The acquircd information can then be used for further process diagnosis.

The pulse-compression method has been used in seismic and acoustic probing applications[2, 3]. This paper considers the application of the method to on-line process monitoring. The principle. the properties of the method, the selection of parameters of the probing signal, and the implementation issues will be dis-

\footnotetext{
1 This work was supported in part by the NASA under Cooperative Agreement \# NCC-1-336, in part by the NSF under Grant \# ECS-9615956, and in part by the Xerox Corporation under Grant \#HE 1321-98.
}

cussed. In addition, its relation to cross-corrclation[5] technique in least squares lincar system identification will be celineated.

\section{Pulse-compression probing method}

Let $u(t)=u_{1}(t)+u_{2}(t)$ be the input signal at one of the input ports of a stable process, where $u_{1}(t)$ is a prescribed set point or a general reference profile, $u_{2}(t)$ is a finite duration probing signal, $y_{1}(t)$ be the response at one of the output ports of the process to $u(t)$ in the absence of $u_{2}(t), y_{2}(t)$ be the process response corresponding to the perturbation by $u_{2}(t), h(t)$ be the iupulse response that reflects the small signal charactcristics of response $y$ to $u$ at the current operating condition, $s(t)$ be a cyclic reference signal, and $z(t)=z_{1}(t)+z_{2}(t)$ be the probing output, where $z_{1}$ and $z_{2}$ correspond to $y_{1}(t)$ and $y_{2}(t)$, respectively. Let '*' denote the convolution operation, and ' $\otimes$ ' denote the correlation operation. Suppose the probing signal $u_{2}(t)$ is suffici nntly small. Then from Fig.1 the following can be seen to hold.

$$
z_{2}(t)=y_{2}(t) \otimes s(t)=h(t) * u_{2}(t) \otimes s(t)=h(t) * e(t),
$$

where $e(t)$ is called an equivalent excitation and is given by

$$
e(t)=u_{2}(t) \otimes s(t) .
$$

If $e(t):=\delta(t)$, then $z_{2}(t)=h(t)$, and the small signal chajacteristics is extracted. In the following development, we attempt to synthesize $e(t)$ by designing signals $u_{2}(t)$ and $s(t)$. Note that $u_{1}(t)$, is fixed by the process speration requirement. The signal design problem amounts to designing $u_{2}(t)$ and $s(t)$ such that the following holds.

$$
y(t) \approx y_{1}(t),
$$

or

$$
\frac{r m s\left\{y_{2}\right\}}{r m s\left\{y_{1}\right\}}
$$

is minimized, and

$$
z(t) \approx z_{2}(t)=h(t)
$$

or

$$
\frac{r m s\left\{z_{2}\right\}}{r m s\left\{z_{1}\right\}}
$$


is maximized. Condition ( 3 ) implies that $u_{2}$ should be constraint in both power and magnitude. The power constraint is dictated by the acceptable signal to noise ratio in response $y(t)$, and the magnitude constraint is dictated by the requirement to cxited the process only in its linear region around the current operating point. Condition (4) is dictated by the acceptable signal to noise ratio in response $z(t)$. Note that the roles of signal and noise are reversed in (3) and in (4). The finite duration requirement on the probing signal $u(t)$ is consistent with the requirement of a small time delay in the probing output that benefits the process monitoring. It is a hard requirement in some applications where the duration is limited, for example, by the interval between the transmitting time and the receiving time of the probing signal.

$u_{2}$ and $s(t)$ satisfying (3) and (4) can be selected to be of the following forms, based on $[2,3]$.

(i) Reference signal $s(t)$ of the correator.

The reference signal is the cyclic repetition of an impulsive $m$-sequence of order $n$ with hit duration $t_{0}$ and intensity 1. An example of an impulsive $m$-sequence $m_{\delta}(t)$ is shown in Fig.2 (b) where $n=5$, resulting a maximum sequence duration of $\left(2^{5}-1\right) \times t_{0}=31 t_{0}$.

(ii) Probing signal $u_{2}(t)$.

$$
u_{2}(t)=p(t) * m_{\delta}(t)
$$

where $p(t)$ is a narrow low pass wavelet of magnitude $A$. Fig.2(a) shows two examples of unity magnitude wavelets. One is a rectangular pulse and the other is a raised cosine pulse. The use of a rectangular wavelet will be assumed from this point on for simplicity. Smoother probing signals such as the $\mathrm{m}$-sequence modulated raiscd cosine is in general more desirable but more complex in signal gencration.

In its original form, a discrete $m$-sequence $m(k)$ of order $n$ and magnitude $a$ is a pseudo-random binary scquence (PRBS) of maximum length $2^{n}-1$ with the following propertics $[4,7]$.

$$
\begin{aligned}
& \left|\frac{1}{2^{n}-1} \sum_{k=1}^{2^{n}-1} m(k)\right|=\frac{a}{2^{n}-1} \\
& \frac{1}{2^{n}-1} \sum_{k=1}^{2^{n}-1} m(k) m(k+l) \\
& = \begin{cases}a^{2}, & k=0, \pm\left(2^{n}-1\right), \pm 2\left(2^{n}-1\right), \\
-\frac{a^{2}}{2^{n}-1}, & \text { elscwherc }\end{cases}
\end{aligned}
$$

Note that the second property holds when at least one of the two $m(k)$ 's involved is cyclically repeated[3].

The probing output is given by

$$
z(t)=y(t) \otimes s(t)=y_{1}(t) \otimes s(t)+y_{2}(t) \otimes s(t) .
$$

The first tcrm in (8) depends on the fixed input and the process. Therefore $y_{1}(t)$ can be assumed to be weakly correlated with $s(t)$. Applying (6), the following inequality can be considered valid

$$
\mid y_{1}\left(t, \otimes s(t) \mid \leq \frac{\max _{t}\left|y_{1}(t)\right| p\left(t-\left(2^{n}-1\right) t_{0}\right)}{\left(2^{n}-1\right) t_{0}} .\right.
$$

Let $T=\left(2^{n}-1\right) t_{0}$. The second term of $(8)$ can be cxpressed in a more detailed manner as

$$
\begin{aligned}
& h(t) * m_{\delta}(t) \otimes s(t) \\
& =h(t) * \frac{1}{T}\left\{2^{n} p(t) * \sum_{i} \delta(t-i T)-p(t) * \sum_{j} \delta\left(t-j t_{0}\right)\right\} .
\end{aligned}
$$

When $n$ is sufficiently large, we have

$$
y_{2}(t) \otimes s(t) \approx h(t) * \frac{p(t-i T)}{t_{0}} .
$$

If in adclition, wavelet $p(t)$ is sufficicntly narrow with respect to the quickest variations in $h(t)$ the above term approxinates $A h(t)$. For the above term to be sufficiently clominant in $z(t)$, it must be sufficiently larger than $\max _{t}\left|y_{1}(t)\right| /\left[\left(2^{n}-1\right) t_{0}\right]$ in $(9)$ which again requires sufficien ly large $n$.

\section{Signil parameters and implementation issues}

Two conditions must be met to arrive at $z(t) \approx A h(t)$. These a:e a sufficiently large $n$ and a sufficiently small $t_{0}$. In this case $z(t)$ is called an estimate of $h(t)$, denoted by $\hat{h}(t)$. This section will discuss in more rigorous terms the meaning of large $n$ and small $t_{0}$.

Recall that $t_{0}$ is the bit duration of the impulsive $m$ sequence in usc. It is also the width of the equivalent excitation $e(t)$ and that of the low-pass wavelet $p(t)$. Therefore $t_{0}$ determines the level of details of $h(t)$ observabic from the probing output $z(t)$, or the resolution of $h_{h}(t)$. In order to observe the full details in $h(t)$, $t_{0}$ should be detcrmined by using Shannon's sampling theorem [1], which states that if the Fourier transform of $h(t)$ is zero outside of $\left(-\omega_{0}, \omega_{0}\right)$, then $h(t)$ can be fully reconstructed based only on its sampled values at a sampling rate higher than $\omega_{s}=2 \omega_{0}$. In practice, $h(t)$ is not strictly band limited, and one may settle with a sampling rate about 5 times the estimated bandwidth $\omega_{B W} /(2 \pi)$ of $h(t)$. This rate translates to the condition

$$
t_{0} \leq 1 / \omega_{B W}
$$

The probing output repeats itself every $T$ seconds. It is obvious that $T$ must be longer than the "memory" of $h(t)$ in order to avoid the time domain aliasing. This would require that knowledge of how long it takes for 
the slowest significant modes of $h(t)$ to decay to approximately zero. A reasonable assessment is $T=5 \tau_{\text {slow }}$ where $\tau_{\text {slow }}$ is the time constant of the slowest mode of $h(t)$. Given $T$ and $t_{0}, n$ can be determined through

$$
n \geq \log _{2}\left(\frac{T}{t_{0}}+1\right)
$$

Therefore, for a specified $T$, a smaller $t_{0}$ (a higher resolution) results in a lager $n$. In practice, however, the above requirement on $n$ is often much less stringent than that imposed by the signal to noise ratio $r m s\left\{z_{2}\right\} / r m s\left\{z_{1}\right\}$. For every unit increment in $n$, this signal to noise ratio is doubled. Another direct benefit for using a larger $n$ is the reduction in bias as shown in (7). Note however, that both a smaller $t_{0}$ and a larger $n$ lead to an increased computational burden in carrying out the cross-correlation. In addition, from the time a probing signal is applied to the time the probing output appears in its entirety (one complete $h(t), 2 T$ must have gone by. Therefore, whericver the signal to noise ratio at $z$ allows, $T$ thus $n$ should be minimized, and whenever the resolution on $\hat{h}$ allows, $t_{0}$ should be maximized.

The pulsc-compression method for process monitoring can be easily implemented digitally, though all the above discussion was carried out in the continuous domain. The digital data storage solves the problem of long delay times normally encountered in using the cross-correlation technique on-line for processes with large time constants[5]. In this case. the impulses in the impulsive $m$-sequence are replaced by pulses, the lowpass wavelet can be replaced by its samples at a sampling rate as low as one sample per bit duration $\left(t_{0}\right)$. The $m$-sequence can be generated by an $n$-stage shift register[4]. The digital signal acquisition and application from and to the process can be accomplished by using $\mathrm{A} / \mathrm{D}$ and $\mathrm{D} / \mathrm{A}$ converters.

\section{Relation to the cross-correlation technique}

The same pseudo-random excitation is used in both the pulsc-compression method for process monitoring introduced in this paper and in the cross-correlation method for impulse response identification for lincar systems casted in least squares formulation $[6,5]$. Therefore the two methods are in fact equivalent, and enjoy the same advantages. Since the methods are nonparametric, little a priory knowledge is required of the process model. Processing and memory requirements are moderate and can be controlled through trading off with the monitoring/identification resolution/accuracy. As long as the process is stable, divergence is not possible. Though not considered in this paper, it has been shown $[5,6]$ that the crosscorrclation method has a superb noise rejection capability. The pulse-compression method differs from the crosi-correlation method in the following aspects. The pulse compression for process monitoring problem is formulated entirely in the continuous domain; the probing signal and the reference signal in our pulsecompresion method are not the same; our method is applicable to nonlinear systems; the analysis is carricd out from a deterministic vicw; the flcxibility of using a generel lowpass wavelct (as opposed to rectangular only) is ntroduced; the unnecessity of the periodic repetition of the probing signal[7] for less noisy process is clarified and therefore reduce the time delay in crosscorrelating. Use of the monitoring data for diagnosis or ident fication purpose is possible[6].

For a cimplete development of the cross-corrclation method using the $m$-sequence for linear system identification, the reader is referred to Hill and McMurtry[5].

\section{An example}

In this scction, the pulse-compression method for process monitoring is applied to a simulated electrohydraulic system that is subject to leakage. The system cxhibits a first order small signal characteristics with a bandvidth of approximately $5 \mathrm{rad} / \mathrm{sec}$. In order to be able to recognize a small percentage change in leakage coefficient, bit duration $t_{0}$ is chosen to be $0.04 \mathrm{sec}$. Using the setup of Fig.1, a continuous probing signal $\left(u_{2}(t)\right)$ une tenth of the magnitude of a windowed sinusoidal signal $\left(u_{1}(t)\right)$ is applied to the system. $u_{2}(t)$ is generated through a 15 th order impulsive $m$-sequence modula1 ed rectangular wavelet. Response $y(t)$ at the system output is shown in Fig.3. The magnitude ratio of $y_{1}$ to $y_{2}$ is about 7. Response $z(t)$ is shown in Fig.4, the marnitude ratio of $z_{2}$ to $z_{1}$ is approximately 40 , yiclding a very accurate estimate of impulse response. This is achieved at the expense of a long delay of approximately $42(=2 T)$ minutes before the cntire impulse response is observed. In practice that the noise level would not necessitate the pursuit of this accuracy, and a shorter delay in acquiring monitoring data is dcsirable.

\section{Conclusions}

A pulse-compression method for on-line monitoring of the smill signal behavior is described. The parameters in the probing signal can be chosen to balance between the speed of processing and the accuracy of the acquired monitoring data, which is the impulse response of the process at the current operating condition. The paper elucidates the concept of stimulating all significant modes of the small signal characteristics of a process through the compression of an elongated small magnitude poring signal of finite duration into 
a near impulsive equivalent excitation signal. Besides the application in process monitoring as discussed in this paper, the pulse-compression nethod has found applications in scismic acquisition[2, 3], and in linear system identification $[5,6,7]$ under a different formalization. Other possible applications include earth crust movement monitoring, ncar surface enginecring applications, paleontologic study, mechanical malfunction in machineries, and biomedical imaging applications.

\section{References}

[1] Aström, J.A., and Wittenmark, B., Computer controlled Systems: Theory and Design, Prcnticc-Hall, 1984.

[2] Chen, H., and Wu, N. E., A method of high resolution and high SNR data acquisition for probing using pulse-compression, US Pat. 5,426,618, 1995.

[3] Chen, H. , and Wu, N. E., Implementation and features of a now practicable pulse-compression probing method- the NZAP method - which can eliminate the intrinsic deficiencies of the conventional method, Proc. SEG 67th Annual Meeting, 1997.

[4] Golomb, S.W., Digital Communication with Space Applications, Prenticc Hall, 1964. Shift Register Sequences, Holden-Day, 1976.

[5] Hill, J.D., and McMurtry, G.J., An application of digital computers to linear system identification, IEEE Trans. on Automatic Control, vol. 19, pp.753768, 1974.

[6] Isermann, R., Baur, U., Bamberger, W., Kneppo, P., and Siebert, H., Comparison of six on-line identification and parameter estimation methods, Automatica, vol.10, pp.81-103, 1974.

[7] Ljung, L., System Identification: the Theory for the User, Prentice-Hall, 1999.

[8] Patton, R.J., , Frank, P.M., and Clark, R.N., (cds.), Issues of Fault Diagnosis for Dynamic Systems, Springer-Verlag, 2000.

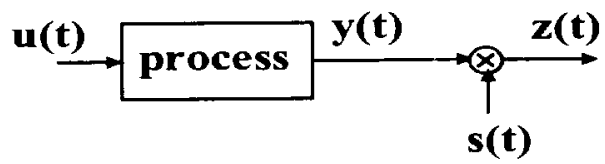

(a)

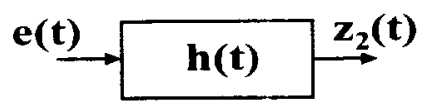

(b)

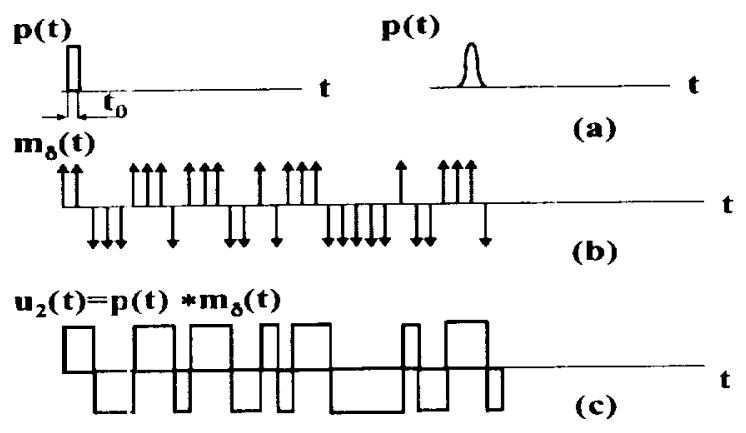

Fig. 2 Lcwpass wavelet examples and a probing signal

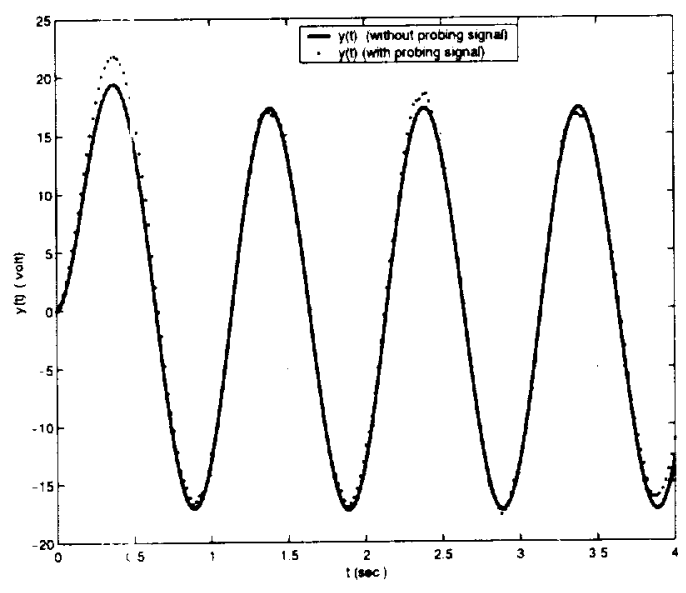

Fig.3 Hydraulic system response to sinusoidal with/without probing signal

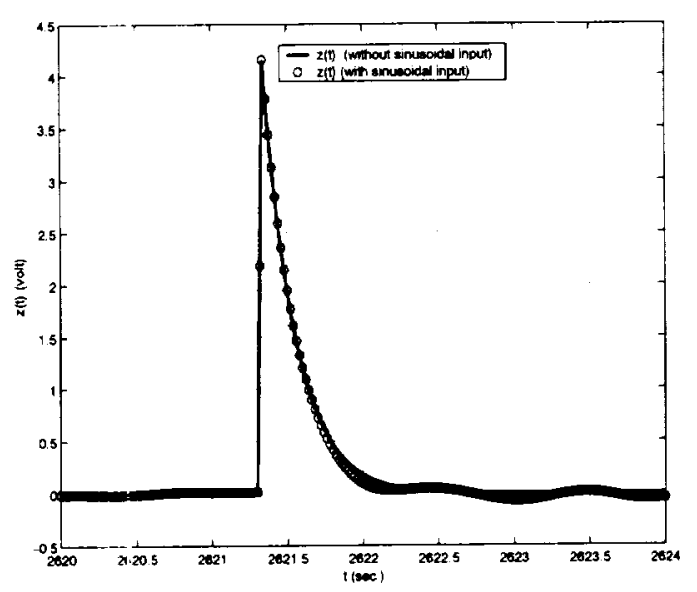

Fig.4 Probing output to probing input with/without sinusoical input

Fig.1 A process monitoring schematic 